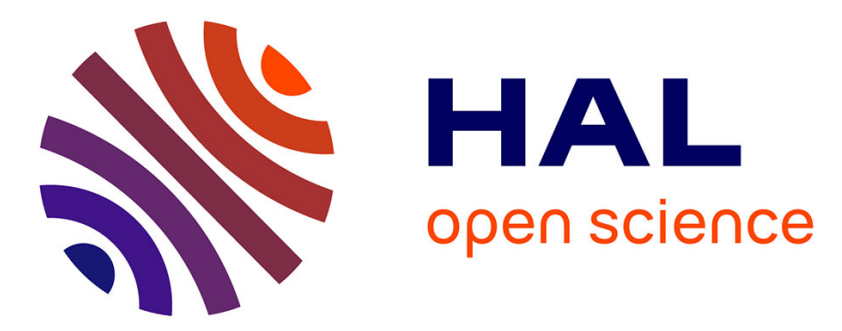

\title{
Dynamics of heavy impurities in non-linear MHD simulations of sawtoothing tokamak plasmas
}

\author{
Jae-H Ahn, Xavier Garbet, H Lütjens, R Guirlet
}

\section{To cite this version:}

Jae-H Ahn, Xavier Garbet, H Lütjens, R Guirlet. Dynamics of heavy impurities in non-linear MHD simulations of sawtoothing tokamak plasmas. 2016. hal-01340291

\section{HAL Id: hal-01340291 \\ https://hal.science/hal-01340291}

Preprint submitted on 30 Jun 2016

HAL is a multi-disciplinary open access archive for the deposit and dissemination of scientific research documents, whether they are published or not. The documents may come from teaching and research institutions in France or abroad, or from public or private research centers.
L'archive ouverte pluridisciplinaire HAL, est destinée au dépôt et à la diffusion de documents scientifiques de niveau recherche, publiés ou non, émanant des établissements d'enseignement et de recherche français ou étrangers, des laboratoires publics ou privés. 


\title{
Dynamics of heavy impurities in non-linear MHD simulations of sawtoothing tokamak plasmas
}

\author{
Jae-H. Ahn, X. Garbet, H. Lütjens* and R. Guirlet \\ CEA, IRFM, F-13108 Saint Paul-lez-Durance, France \\ ${ }^{*}$ Centre de Physique Théorique, Ecole Polytechnique, CNRS, F-91128 Palaiseau, \\ France \\ E-mail: jae-heon.ahn@polytechnique.edu \\ Draft : 22 June 2016
}

\begin{abstract}
.
The effect of sawteeth on impurity dynamics is studied with the XTOR-2F code. Non-linear full 3D MHD simulations including appropriate fluid equations for heavy impurities show that the presence of regular sawtooth crashes affects the impurity behaviour. A spatial non-uniformity of $5 \%$ in post-crash impurity density profiles persists due to 2D structures of impurity density which appear during sawtooth crashes. They are shown to be mainly driven by the $\mathbf{E} \times \mathbf{B}$ velocity, and are responsible for the sudden impurity transport in the core plasmas.
\end{abstract}

\section{Introduction}

Understanding the dynamics of heavy impurities in fusion plasmas is an important issue since it has been decided to equip Iter with a tungsten divertor. Tungsten has the great advantage of high heat flux handling capability, low erosion and low tritium retention [1]. However, metallic impurities in the core not only dilute the plasma, but may also lead to a radiative collapse due to excessive cooling, even when they are present in small concentration [2],[3]. Preventing excessive impurity influxes by means of auxiliary heating is an active subject of research [4],[5],[6],[7].

In the core region of present tokamaks, heavy impurities are highly collisional due to their high charge number. Also, neoclassical transport is enhanced for heavy impurities due to the poloidal asymmetry of the impurity density caused by the centrifugal force [8],[9],[10]. Indeed, the effects due to rotation are stronger for heavy impurities. For heavy impurities, the Mach number $M_{z}$ can be of order unity $\left(M_{z} \sim 1\right)$ even if $M_{i} \ll 1$ for the main ion species. As a consequence, neoclassical transport has to be considered along with turbulent transport for heavy impurities. For high collision frequencies, the relevant framework is the Pfirsch-Schlüter regime. However, the main ion species, with a single charge for deuterium and tritium, remains in the low collisional banana or plateau regime in the core region. In this case, neoclassical theory predicts that the 
ion temperature gradient drives an outward impurity flux, and is thus responsible for a favourable thermal screening effect [11],[12]. Recent experiments and simulations with transport codes are consistent with this picture [13],[14]. In addition, strong toroidal rotation is shown to affect the impurity transport properties. Indeed, the impurity diffusivity is enhanced with toroidal plasma rotation [15]. Also, the thermal screening effect is weakened due to the effect of rotation, and can even change its sign so that the temperature gradient contribution drives an unfavourable influx of impurity [16],[17]. For this study, the toroidal rotation is considered to be small so that this unfavourable effect due to the centrifugal force is neglected.

In addition, magneto-hydrodynamic (MHD) instabilities such as tearing modes and sawtooth oscillations add complexity to the impurity behaviour in core plasmas. Indeed, it is observed that the presence of tearing modes locally increases the impurity diffusivity in the islands and enhances accumulation [18]. Also, discrepancies are found between the measured impurity behaviour in presence of the internal kink mode and the neoclassical and turbulent predictions [19]. It is well known that sawtooth crashes drive an efficient core radial transport of impurities [20],[21]. If the pre-crash impurity profile is peaked, the profile is flattened after a sawtooth crash in a region which is approximatively encompassed by the $q=1$ surface. On the contrary, for a hollow impurity density profile, the impurities penetrate inside the $q=1$ surface. This behaviour is consistent with the Kadomtsev reconnection model [23],[24] for different pre-crash impurity profiles [25]. In addition, the presence of 2D structures of impurity density during sawtooth crashes has been evidenced in the XTOR-2F simulations [26].

This paper illustrates and analyses heavy impurity transport during sawtooth oscillations with the non-linear full 3D MHD XTOR-2F code [27]. The XTOR-2F code is now generalized by the inclusion of an impurity continuity equation and a parallel impurity momentum equation. Both are coupled self-consistently with the two-fluid set of equations describing the bulk plasma, which is an important improvement compared to the work in Ref.[26]. In Section 2, it is shown that the Pfirsch-Schlüter flux is recovered when using the appropriate fluid equations for impurity. A brief description of the non-linear MHD model in the XTOR-2F code and numerical settings used for the present work is presented in Section 3. The outputs of the XTOR-2F code are illustrated and analysed in Section 4. First, it is shown that the expected neoclassical impurity fluxes are reproduced with the implemented fluid equations for impurity. Second, the presence of sawteeth is found to modify deeply the impurity dynamics compared to the predictions from neoclassical theory. Section 5 is dedicated to a discussion on the dynamics of the 2D impurity density structures during sawtooth crashes, before concluding in Section 6 . 


\section{Fluid model for highly collisional impurities}

\subsection{Orderings}

A species is in a highly collisional regime when the collisionality $\bar{\nu} \equiv \nu / \omega_{t} \gg 1$, where $\nu$ and $\omega_{t}$ are the characteristic collision and transit frequencies respectively. The distribution function in the velocity space is then relaxed to a Maxwellian function due to the collisions. Thus, the fluid description is legitimate in a collisional regime. MHD equations are derived from the kinetic equation by supposing a large perpendicular electric field, i.e. $\left|\mathbf{V}_{E}\right| / V_{T} \sim 1$, where $\mathbf{V}_{E}=\mathbf{E} \times \mathbf{B} / B^{2}$ is the $\mathbf{E} \times \mathbf{B}$ velocity and $V_{T}$ is the thermal velocity. Here, $\mathbf{E}$ and $\mathbf{B}$ represent respectively the electric and magnetic fields and $B=|\mathbf{B}|$. In the framework of the neoclassical theory, rapid fluid motions are neglected by using the drift ordering where $\left|\mathbf{V}_{E}\right| / V_{T} \sim \rho^{*}$. Here, the parameter $\rho^{*} \equiv \rho_{L} / L \ll 1$, where $\rho_{L}$ is the Larmor radius and $L$ a macroscopic scale length.

The beginning points are the continuity and momentum equations for the species $a$ in the Pfirsch-Schlüter regime

$$
\begin{aligned}
& \partial_{t} N_{a}+\boldsymbol{\nabla} \cdot\left(N_{a} \mathbf{V}_{a}\right)=0 \\
& N_{a} m_{a}\left(\partial_{t} \mathbf{V}_{a}+\left(\mathbf{V}_{a} \cdot \boldsymbol{\nabla}\right) \mathbf{V}_{a}\right)=-\boldsymbol{\nabla} P_{a}+N_{a} e_{a}\left(\mathbf{E}+\mathbf{V}_{a} \times \mathbf{B}\right)+\boldsymbol{\mathcal { R }}_{a}
\end{aligned}
$$

Here, $e_{a}, N_{a}, m_{a}$ and $P_{a}$ are respectively the charge, density, mass and pressure of the species $a$. The pressure anisotropy is neglected since high collisionality is considered for this study [28]. The velocity of the species $a$ is a sum of the $\mathbf{E} \times \mathbf{B}$, diamagnetic and parallel velocities

$$
\mathbf{V}_{a}=\mathbf{V}_{E}+\mathbf{V}_{a}^{*}+V_{\|, a} \mathbf{B} / B
$$

where $\mathbf{V}_{a}^{*}=\mathbf{B} \times \nabla P_{a} /\left(N_{a} e_{a} B^{2}\right)$. In this paper, the index $\|$ represents a projection along the unit vector $\mathbf{B} / B$.

In the force balance equation, the effect of collisions between the species $a$ and other species $b$ lies in the friction force $\boldsymbol{\mathcal { R }}_{a}=\sum_{b} \boldsymbol{\mathcal { R }}_{a b}$. The friction force between two species $\boldsymbol{\mathcal { R }}_{a b}$ is a sum of drag and thermal forces. A drag force is induced by a difference of velocities between the two species. A thermal force appears due to the velocity dependence of collision frequencies and is proportional to the parallel heat fluxes. From the action-reaction principle, $\boldsymbol{\mathcal { R }}_{a b}=-\boldsymbol{\mathcal { R }}_{b a}$. As neoclassical fluxes are associated with parallel friction, the parallel components of the friction force are to be expressed in terms of different gradient lengths in the incompressible fluid limit.

In the drift ordering where flow variations are slow, the force balance equation (2) reads

$$
N_{a} e_{a}\left(\mathbf{E}+\mathbf{V}_{a} \times \mathbf{B}\right)=\nabla P_{a}-\boldsymbol{\mathcal { R }}_{a}
$$

Through this study, the inertia term $\left(\mathbf{V}_{a} \cdot \boldsymbol{\nabla}\right) \mathbf{V}_{a}$ is supposed to be small compared to other terms in the expression (4). In other words, the toroidal plasma rotation is considered to be weak enough so that the centrifugal force can be neglected. 
Tokamak plasmas are usually magnetized with a field such that the gyrofrequency is large compared to the collision frequency. This is described by the small parameter $\delta \equiv \nu / \omega_{c} \ll 1$, where $\omega_{c}$ is the cyclotron frequency. Each field is then separated into a mean field represented by a flux surface averaged quantity and small perturbations which correspond to poloidal asymmetries. For example, the density $N$ in the toroidal coordinate system $(\psi, \theta, \varphi)$ is written as $N(\psi, \theta)=N_{0}(\psi)+N_{1}(\psi, \theta)+O\left(\delta^{2}\right)$ with $\left|N_{1}(\psi, \theta)\right| / N_{0}(\psi) \sim O(\delta) \ll 1$

It should be noted that neoclassical theory is derived assuming that the plasma is in a quiescent state, i.e. in a configuration of nested magnetic flux surfaces for long time scales. These assumptions are likely not fulfilled during a sawtooth crash. However, this is not a strong limitation since particle and heat fluxes are mainly tied to fast reconnection processes during the crash.

\subsection{Radial particle flux driven by parallel forces}

In the toroidal coordinate system $(\psi, \theta, \varphi)$, the magnetic field $\mathbf{B}$ is written in the form

$$
\mathbf{B}=I(\psi) \boldsymbol{\nabla} \varphi+\boldsymbol{\nabla} \varphi \times \boldsymbol{\nabla} \psi
$$

where the toroidal component $I$ represents also the poloidal current flux function. With the expression (5) of the magnetic field, the projection of the equation (4) along the toroidal direction $R^{2} \nabla \varphi$, where $R$ is the major radius, reads [29]

$$
N_{a} e_{a} \mathbf{V}_{a} \cdot \boldsymbol{\nabla} \psi=-R^{2} \mathcal{R}_{a} \cdot \boldsymbol{\nabla} \varphi
$$

Here, the quantities are supposed to be axisymmetric : $\partial_{\varphi} P_{a}=0$ and $\partial_{\varphi} \phi=0$ with $\phi$ the electric potential. Also, the ion and impurity Ware fluxes are negligible compared to neoclassical fluxes (for more detail, see p.1155 of Ref.[11]) so that the contribution of the induction field to $E_{\varphi}$ can be ignored. Thus, $E_{\varphi} \simeq 0$ under these conditions.

Neoclassical transport is associated with poloidal asymmetries along nested magnetic flux surfaces. The neoclassical impurity particle flux across the flux surfaces is then $\Gamma_{a}^{\psi}=\left\langle\boldsymbol{\Gamma}_{a} . \boldsymbol{\nabla} \psi\right\rangle$, where

$$
\langle G\rangle \equiv \int \frac{G \mathrm{~d} \theta}{\mathbf{B} \cdot \boldsymbol{\nabla} \theta} / \int \frac{\mathrm{d} \theta}{\mathbf{B} \cdot \nabla \theta}
$$

corresponds to flux surface average of the quantity $G$. By writing $\boldsymbol{\mathcal { R }}_{a}=\mathcal{R}_{\|, a} \mathbf{B} / B+\boldsymbol{\mathcal { R }}_{\perp, a}$,

$$
\Gamma_{a}^{\psi}=-\frac{I(\psi)}{e_{a}}\left\langle\frac{1}{B} \mathcal{R}_{\|, a}\right\rangle
$$

The perpendicular friction force $\boldsymbol{\mathcal { R }}_{\perp, a}$ is responsible for the classical transport. It is neglected since the classical flux is small compared to the neoclassical flux at large aspect ratio [29]. The parallel friction force $\mathcal{R}_{\|, a}$ is to be written as function of gradient lengths to derive the expression of the neoclassical radial flux.

For this study, the plasma is supposed to contain a single type of impurity ion, and the impurity is considered as a trace $\left(\alpha_{z} \equiv N_{z} e_{z}^{2} /\left(N_{i} e_{i}^{2}\right) \ll 1\right)$. For heavy impurities 
$\left(m_{z} / m_{i} \gg 1\right)$, the friction force $\boldsymbol{R}_{z} \simeq \boldsymbol{\mathcal { R }}_{z i}$ and its projection along the parallel direction is then written in the form [12],[30],[31]

$$
\mathcal{R}_{\|, z i}=-m_{z} N_{z} \nu_{z i}\left(V_{\|, z}-V_{\|, i}\right)-\frac{2}{5} C_{0} m_{z} N_{z} \nu_{z i} \frac{Q_{\|, i}}{P_{i}}
$$

Here, $Q_{\|, i}$ is the parallel heat flux of main ion species and $\nu_{z i}$ the collision frequency between impurity and main ion species. A general expression of the collision frequency between two species $a$ and $b$ is

$$
\nu_{a b}=\frac{4 \sqrt{2 \pi}}{3} \frac{N_{b}}{m_{a}}\left(\frac{1}{m_{a}}+\frac{1}{m_{b}}\right) \frac{e_{a}^{2} e_{b}^{2} \Lambda}{\left(4 \pi \epsilon_{0}\right)^{2}} \frac{1}{\left(V_{T, a}^{2}+V_{T, b}^{2}\right)^{3 / 2}}
$$

where $\Lambda$ is the Coulomb logarithm.

Ref.[31] adopted Braginskii's procedure for electron-ion collision [30] to derive the impurity flux in the Pfirsch-Schlüter regime. This computation considers both the impurity and the main ion species in the Pfirsch-Schlüter regime. In this case, the favourable thermal screening effect predicted by the neoclassical theory is not retrieved. Hence, the coefficient $C_{0}$ is an adjustable parameter, which is chosen in order to obtain the appropriate screening factor for the impurity Pfirsch-Schlüter flux with bulk ions in the low collisional regime. In general, $C_{0}$ depends on the impurity strength parameter $\alpha_{z}$, and is thus a radial function which is deduced from kinetic computations accounting for collisions. Since this study deals with trace impurities only $\left(\alpha_{z} \ll 1\right), C_{0}$ can be chosen as a constant.

\subsection{Derivation of impurity radial flux}

It is shown in the expression (8) that the perpendicular Pfirsch-Schlüter flux is associated with the parallel friction force. Hence, parallel velocities and heat fluxes have to be derived in order to deduce $\mathcal{R}_{\|, z i}$ according to the expression (9). For this, the assumption on fluid incompressibility is crucial. The perpendicular particle and heat flows are then balanced by parallel flows to conserve particles and heat.

At the lowest order in $\delta$ and using the expressions (3) and (5), the impurity flux of species $a$ reads

$$
\boldsymbol{\Gamma}_{a}=K_{a}(\psi, \theta) \mathbf{B}-\frac{P_{a, 0}}{e_{a}} A_{P_{a}^{*}, \psi} R^{2} \nabla \varphi
$$

where

$$
A_{P_{a}^{*}, \psi} \equiv \partial_{\psi} \ln P_{a, 0}+\frac{e_{a}}{T_{a, 0}} \partial_{\psi} \phi_{0}
$$

and $K_{a}$ is a priori a function of $\psi$ and $\theta$. In the neoclassical time scale, the continuity equation

$$
\partial_{t} N_{a}+\nabla \cdot \Gamma_{a}=0
$$

yields the fluid incompressibility equation $\boldsymbol{\nabla} \cdot \boldsymbol{\Gamma}_{a}=0$ for each species $a$. In this constraint, $K_{a}$ needs to be a radial function. As a consequence, the parallel velocity of the species $a$ is written as

$$
V_{\|, a}=\frac{K_{a}(\psi) B}{N_{a, 0}}-\frac{T_{a, 0}}{e_{a}} \frac{I(\psi)}{B} A_{P_{a}^{*}, \psi}
$$


Unlike for the particle flux $\boldsymbol{\Gamma}_{a}$, the assumption $\boldsymbol{\nabla} \cdot \mathbf{Q}_{a}=0$ for each species $a$, is not general. Indeed, the energy equation for the species $a$ is written in the form

$$
\partial_{t}\left(N_{a} \mathcal{S}_{a}\right)+\nabla \cdot\left(N_{a} \mathcal{S}_{a} \mathbf{V}_{a}\right)=-\frac{2}{3} \frac{\nabla \cdot \mathbf{Q}_{a}}{T_{a}}+\sum_{b} \mathcal{H}_{a b}
$$

where $\mathcal{S}_{a} \equiv \ln \left(P_{a} N_{a}^{-\gamma}\right)$ is the entropy, and $\mathcal{H}_{a b}$ is the energy exchange with another species $b$. Hence, even if the convective term is negligible, only the total heat flux is divergence free (i.e. $\nabla \cdot \sum_{a} \mathbf{Q}_{a}=0$ ). Nevertheless, $\nabla \cdot \mathbf{Q}_{a}=0$ for each species $a$, if the energy exchanges due to collisions with other species $\sum_{b} \mathcal{H}_{a b}$ are negligible. This is the case for species with large mass disparity, as the collision operator is proportional to the mass ratio. In the case of plasmas with heavy impurities, main ions and electrons, this assumption is valid.

The heat flux at the lowest order in $\delta$ reads

$$
\mathbf{Q}_{a}=L_{a}(\psi) \mathbf{B}-\frac{5}{2} \frac{P_{a, 0}}{e_{a}} \partial_{\psi} T_{a, 0} R^{2} \nabla \varphi
$$

where $L_{a}$ is constant along magnetic flux surfaces because of the incompressible heat flux constraint $\boldsymbol{\nabla} \cdot \mathbf{Q}_{a}=0$. Therefore, the parallel heat flux has the following form

$$
Q_{\|, a}=L_{a}(\psi) B-\frac{5}{2} \frac{P_{a, 0}}{e_{a}} \frac{I(\psi)}{B} \partial_{\psi} T_{a, 0}
$$

With the expressions (14) and (17), the parallel friction force $\mathcal{R}_{\|, z i}$ manifests as a function of different radial derivatives. The force balance equation (4) projected along the parallel direction of the impurity $z$ gives

$$
N_{z} e_{z} \nabla_{\|} \phi=\nabla_{\|} P_{z}-\mathcal{R}_{\|, z i}
$$

Averaging Eq.(18) over flux surfaces, with $N_{z} \simeq N_{z, 0}(\psi)$, gives the constraint

$$
\left\langle B \mathcal{R}_{\|, z i}\right\rangle=0
$$

by definition of flux-surface average operator in Eq.(7). From the expressions (9), (14) and (17), the perpendicular impurity particle flux then reads

$$
\begin{aligned}
\Gamma_{z}^{\psi}=-m_{z} N_{z, 0} T_{0} \nu_{z i} \frac{I(\psi)^{2}}{e_{z}^{2}}\left[\left\langle\frac{1}{B^{2}}\right\rangle-\frac{1}{\left\langle B^{2}\right\rangle}\right] \\
\left(\partial_{\psi} \ln P_{z, 0}-\frac{e_{z}}{e_{i}} \partial_{\psi} \ln P_{i, 0}+C_{0} \frac{e_{z}}{e_{i}} \partial_{\psi} \ln T_{i, 0}\right)
\end{aligned}
$$

by considering the isothermal assumption $T_{z} \simeq T_{i}=T$.

For a geometry of circular concentric magnetic surfaces, it is known [28] that

$$
I(\psi)^{2}\left[\left\langle\frac{1}{B^{2}}\right\rangle-\frac{1}{\left\langle B^{2}\right\rangle}\right] \simeq 2 r^{2}
$$

in the limit of small inverse aspect ratio $\varepsilon \equiv r / R_{0} \ll 1$, where $r$ and $R_{0}$ are respectively the minor and major radii of a flux surface. Also, $\partial_{r} \psi=B_{0} r / q$ in this approximation, with $q$ the safety factor. For heavy impurities, $e_{z} / e_{i} \gg 1$ and the radial flux reads

$$
\begin{aligned}
\left\langle\boldsymbol{\Gamma}_{z} \cdot \nabla r\right\rangle & =\left\langle\frac{q}{B_{0} r} \Gamma_{z}^{\psi}\right\rangle \\
& =-D_{z}^{P S} N_{z, 0}\left\{\partial_{r} \ln N_{z, 0}-\frac{e_{z}}{e_{i}} \partial_{r} \ln N_{i, 0}+\left(C_{0}-1\right) \frac{e_{z}}{e_{i}} \partial_{r} \ln T_{i, 0}\right\}
\end{aligned}
$$


with the Pfirsch-Schlüter diffusion coefficient $D_{z}^{P S}=2 \rho_{z}^{2} q^{2} \nu_{z i}$ and the impurity Larmor radius $\rho_{z}=\sqrt{m_{z} T_{z, 0}} /\left(e_{z} B_{0}\right)$.

Depending on the value of the coefficient $C_{0}$ in the expression (22), the temperature gradient will reduce or enhance the impurity influx. For trace impurities (i.e. $\alpha_{z} \ll 1$ ), computations from the neoclassical theory show that $C_{0} \simeq 1$ when the impurity and the main ion species are both in the Pfirsch-Schluter regime [31]. In that case, the temperature gradient has no effect on the impurity radial flux. However, for the most common case of heavy impurities in the Pfirsch-Schlüter regime and the main ion species in the banana-plateau regime, the coefficient $C_{0}$ is predicted to be $C_{0}=3 / 2$ in Ref.[11]. The ion temperature gradient then drives a favourable thermal screening effect. For this study, $C_{0}=3 / 2$ is set in the expression (9) of the collisional force to reproduce the appropriate neoclassical prediction.

The derivation of the Pfirsch-Schlüter fluxes in this section is done under the assumption of small poloidal asymmetries compared to surface averaged quantities. It is shown in Appendix A that the relative level of poloidal asymmetry of the impurity density $N_{z, 1} / N_{z, 0}$ is proportional to the parameter $\delta \ll 1$. However, the assumption of small poloidal asymmetries is far from being satisfied. Indeed, $N_{z, 1} / N_{z, 0}$ is also proportional to the square of the safety factor $q^{2}$, the impurity charge and the ion density and temperature gradient lengths. Therefore, the assumption becomes easily invalid in the edge region, where the safety factor values can go up to $q \simeq 5$ and the ion density and temperature gradients are steep. In the core region, $q \simeq 1$ and the ion density and temperature gradient lengths are usually large. Hence, the validity condition is fulfilled in the framework of this study.

\section{Numerical methods}

\subsection{Non-linear MHD model in the XTOR-2F code}

The XTOR-2F code [27] is a non-linear and two-fluid full 3D MHD code. The following set of fluid equations is solved with a Newton-Krylov method using a fully implicit scheme :

$$
\begin{aligned}
& \partial_{t} N+\nabla .(N \mathbf{V})+\left(\nabla \times \frac{\mathbf{B}}{B^{2}}\right) \cdot \frac{\nabla P_{i}}{e_{i}}=\nabla \cdot\left(D_{\perp} \boldsymbol{\nabla} N\right)+S_{N} \\
& N m_{i}\left(\partial_{t} \mathbf{V}+(\mathbf{V} . \boldsymbol{\nabla}) \mathbf{V}+\left(\mathbf{V}_{i}^{*} . \boldsymbol{\nabla}\right) \mathbf{V}_{\perp}\right)=\mathbf{J} \times \mathbf{B}-\boldsymbol{\nabla} P+\mu_{i} \nabla^{2}\left(\mathbf{V}+\mathbf{V}_{i}^{*}\right) \\
& \partial_{t} P+\mathbf{V} \cdot \boldsymbol{\nabla} P+\gamma P \boldsymbol{\nabla} \cdot \mathbf{V}+\frac{\gamma}{e_{i}}\left(\boldsymbol{\nabla} \times \frac{\mathbf{B}}{B^{2}}\right) \cdot\left(T \nabla P_{i}+P_{i} \boldsymbol{\nabla} T_{i}+P_{e} \boldsymbol{\nabla} T_{e}\right) \\
& =(\gamma-1)\left[\nabla \cdot\left(N \chi_{\|} \nabla_{\|} T \mathbf{b}\right)+\nabla \cdot\left(N \chi_{\perp} \nabla_{\perp} T\right)\right]+S_{H} \\
& \partial_{t} \mathbf{B}=\boldsymbol{\nabla} \times(\mathbf{V} \times \mathbf{B})+\nabla \times\left(\frac{\nabla_{\|} P_{e}}{N e_{i}} \mathbf{b}\right)-\nabla \times(\eta \mathbf{J})
\end{aligned}
$$

Here, the indices $i$ and $e$ designate the ion and electron populations, $\mathbf{V} \equiv \mathbf{V}_{E}+\mathbf{V}_{\|, i}$ is the plasma fluid velocity, $P=P_{e}+P_{i}$ is the total pressure, $S_{N}$ and $S_{H}$ are the 
particle and heat sources, $\gamma$ is the heat capacity ratio, $D_{\perp}$ is the perpendicular particle diffusion coefficient, $\chi_{\|}$and $\chi_{\perp}$ are the parallel and perpendicular heat diffusivities, $\eta$ is the plasma resistivity and $\mu_{i}$ is the plasma viscosity. Also, $N=N_{e}=N_{i}$ due to the quasi-neutrality constraint in the trace impurity limit. The total temperature is defined as $T=P / N$. The resistivity $\eta$ varies in time with the plasma temperature, following the Spitzer's resistivity model $\eta \propto T^{-3 / 2}$.

In addition, fluid equations for impurity are implemented in the XTOR-2F code and coupled with other MHD equations

$\partial_{t} N_{z}+\nabla \cdot\left(N_{z} \mathbf{V}_{z}\right)=\boldsymbol{\nabla} \cdot\left(D_{\perp, z} \boldsymbol{\nabla} N_{z}-\mathbf{V}_{p, z} N_{z}\right)+S_{z}$

$N_{z} m_{z}\left(\partial_{t} V_{\|, z}+\left(\mathbf{V}_{z} \cdot \nabla\right) V_{\|, z}\right)=-\nabla_{\|} P_{z}-N_{z} e_{z} E_{\|}-\mathcal{R}_{\|, z i}$

where $\mathbf{V}_{z}$ is the impurity fluid velocity as defined in (3), $D_{\perp, z}$ and $\mathbf{V}_{p, z}$ are the perpendicular impurity diffusion coefficient and radial pinch velocity, $S_{z}$ is the impurity density source, and $\mathcal{R}_{\|, z i}$ is the parallel friction force represented in (9).

The XTOR-2F code uses a finite difference method in the radial direction, and a pseudo-spectral method in the poloidal and toroidal directions. Time-steps are automatically adjusted for an optimum number of iterations for the Newton-Krylov method. The plasma dynamics such as sawtooth crashes, which occur during a very short time scale compared to the confinement time, can then be studied with an appropriate time resolution [32],[26]. The radial resolution is chosen such that the current sheet at the reconnection layer is accurately computed. The mesh used in the simulations is $\left(M_{r}, M_{\theta}, M_{\phi}\right)=(255,32,12)$, where $M_{r}, M_{\theta}, M_{\phi}$ are respectively the number of grid points in $r, \theta, \phi$ directions. A total of 4 toroidal modes $(n=0, \ldots, 3)$ and $n+8$ poloidal modes $(m=0, \ldots, n+7)$ for each toroidal mode, are evolved in the simulations. A discussion on the mode resolution used in the XTOR-2F simulations to describe sawtooth cycles can be found in Refs.[27],[33],[34].

\subsection{Plasma parameter settings and initial conditions}

The plasma parameters used in the simulations are rescaled to avoid excessive simulation times. Especially, the Lundquist number in tokamaks of the order $S \sim 10^{8}-10^{9}$ gives large resistive times, which is numerically demanding. Hence, the Lundquist number is reduced to $S=10^{7}$ for the present XTOR-2F sawtooth simulations. Other plasma parameters are rescaled so as to preserve the ratios between different characteristic time scales with respect to experimental values. The diamagnetic parameter is then set to $\bar{d}_{i}=0.1$, which is about two or three times larger than the experimental values. Also, the normalized perpendicular heat diffusivity is set to $\bar{\chi}_{\perp}=3 \times 10^{-6}$. The products $S \bar{d}_{i}$ and $S \bar{\chi}_{\perp}$ are then about a third of those expected from the experiments. As far as the viscosity is concerned, measurements of this quantity in experiments are not available. In this paper, the normalized plasma viscosity is set to $\bar{\mu}=5 \times 10^{-6}$, so that the magnetic Prandtl number is $P r_{m}=S \bar{\mu}=50$. 


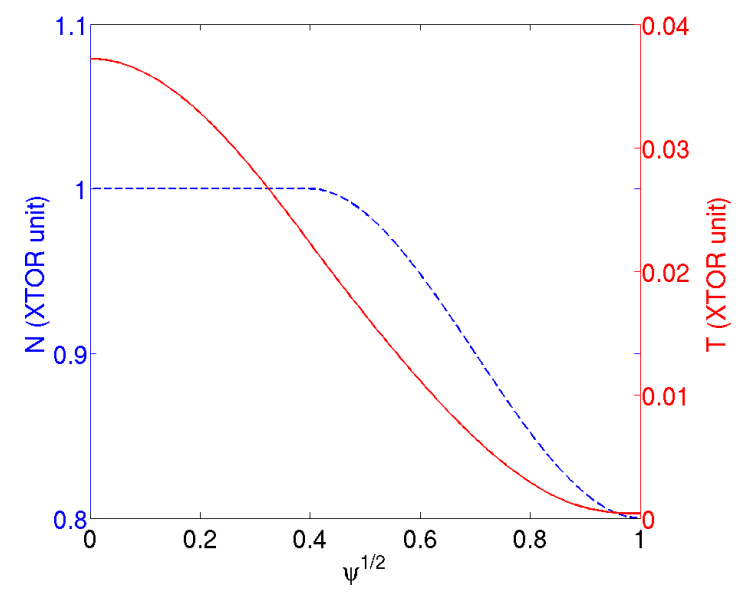

Figure 1. The initial density (dashed line) and temperature (solid line) profiles used in the simulations presented in this paper.

Note that the physical times and the physical lengths are normalized respectively to the Alfvén time $\tau_{A}$ and the minor radius $a$. The parameter $\tau \equiv P_{i} / P_{e}$ becomes $\tau=T_{i} / T_{e}$ under the quasi-neutrality constraint $N_{e}=N_{i}$. This parameter thus accounts for the difference between the electron and ion temperatures. In the following simulations, electron and ion temperatures are equal, i.e. $\tau=1$. The initial density and temperature profiles used for the present study are shown in Fig.1. For the following simulations, a flat initial impurity density profile is set. The main ion density is normalized to the onaxis density value $N_{0}=3 \times 10^{19} \mathrm{~m}^{-3}$. The temperature is normalized to $a^{2} B_{0}^{2} /\left(R_{0}^{2} N_{0} \mu_{0}\right)$ where the values of the on-axis magnetic field $B_{0}$ and the major radius $R_{0}$ are taken at geometric axis. The normalized impurity density is $\bar{N}_{z}=1$ with $\bar{N}_{z} \equiv N_{z} / N_{z, 0}$ and $N_{z, 0}=10^{-5} N_{0}$.

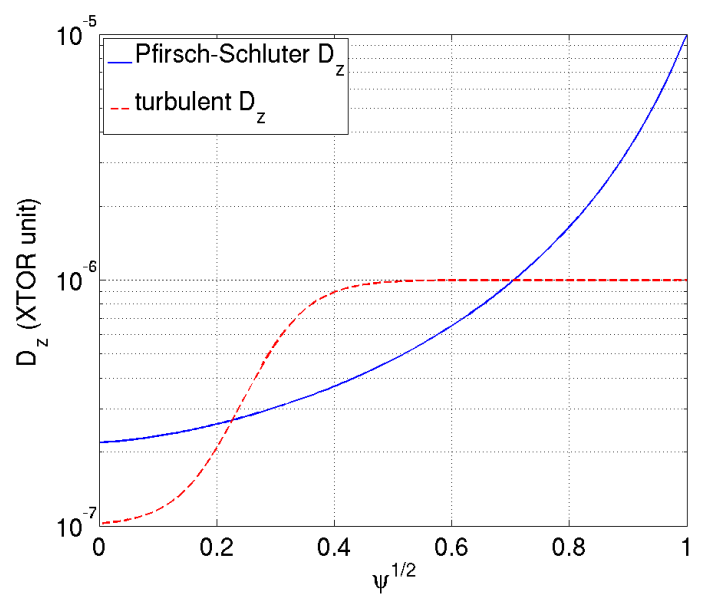

Figure 2. The initial diffusion profile set to represent the turbulent transport (dashed line), and the initial Pfirsch-Schlüter diffusion profile (solid line) computed intrinsically from the impurity fluid equations. 
In the present XTOR-2F model, the transport coefficients $D_{\perp, z}$ and $\mathbf{V}_{p, z}$ on the right hand side of the impurity density equation (28) include the contributions of turbulent, classical and neoclassical transport. However, calculations of Section 2 show that a Pfirsch-Schlüter impurity flux is driven by poloidal asymmetries. Thus, it is important to notice that the Pfirsch-Schlüter particle flow is intrinsically present in the $\boldsymbol{\nabla} \cdot \boldsymbol{\Gamma}_{z}=\boldsymbol{\nabla} \cdot\left(N_{z} \mathbf{V}_{z}\right)$ term of the Eq.(28). Hence, the coefficients $D_{\perp, z}$ and $\mathbf{V}_{p, z}$ do not contain the Pfirsch-Schlüter coefficients. In Fig.2, the red dashed line represents the diffusion coefficient $D_{\perp, z}$ on the right hand side of Eq.(28) which is set to reproduce the experimental radial shape. Indeed, this diffusion coefficient profile is based on fluctuation measurements which show that turbulence intensity is small inside the $q=1$ surface [35],[36]. The blue solid line in Fig.2 corresponds to the Pfirsch-Schlüter diffusion coefficient computed from the poloidal asymmetries as shown in Section 2. It can be noticed that the proportionality to $q^{2}$ of the diffusion coefficient in the PfirschSchlüter regime is recovered in the limit of $\epsilon=r / R_{0} \ll 1$ as shown by Eq.(22). The collision frequency $\nu_{z i}$ is chosen such that the Pfirsch-Schlüter and 'turbulent' diffusion coefficients are of the same order of magnitude in the core region. The neoclassical impurity pinch velocity in the Pfirsch-Schlüter regime, depending on the main ion density and temperature gradient lengths, is taken into account by the model. However, the 'turbulent' pinch velocity $\mathbf{V}_{p, z}$ on the right hand side of Eq.(28) is set to zero in this study. In the simulations, fully-stripped carbon $\left(C^{6+}\right)$ is chosen as the impurity species.

\section{Simulation results}

\subsection{Validation of the implemented model}

It was shown in Section 2 that the Pfirsch-Schlüter impurity flux is related to the collisional force $\boldsymbol{\mathcal { R }}_{z i}$ between the main ion species and the impurity. Moreover, the expression of the impurity radial flux in Eq.(22) shows that the drag force and the thermal force give respectively the appropriate contributions of the main ion density gradient and the temperature gradient. In a first step, simulations without the internal kink mode are performed in order to verify whether the implemented model reproduces the radial impurity flux, as expected.

To illustrate this point, cases with and without thermal force are simulated by setting appropriate values of the coefficient $C_{0}$ in the expressions (9) and (22). With the density and temperature profiles presented in Fig.1, an influx of impurity is obtained by considering only the drag force $\left(C_{0}=0\right)$. For the case with thermal force, the coefficient $C_{0}$ is set to $C_{0}=3 / 2$, so that an outward flux of impurity appears due to the temperature screening effect, as predicted in Ref.[11]. In Fig.3, the time evolution of the flux surface average of the impurity density profile is presented for the two cases. These figures show that the impurity radial fluxes behave in accordance with the analytical calculations. 

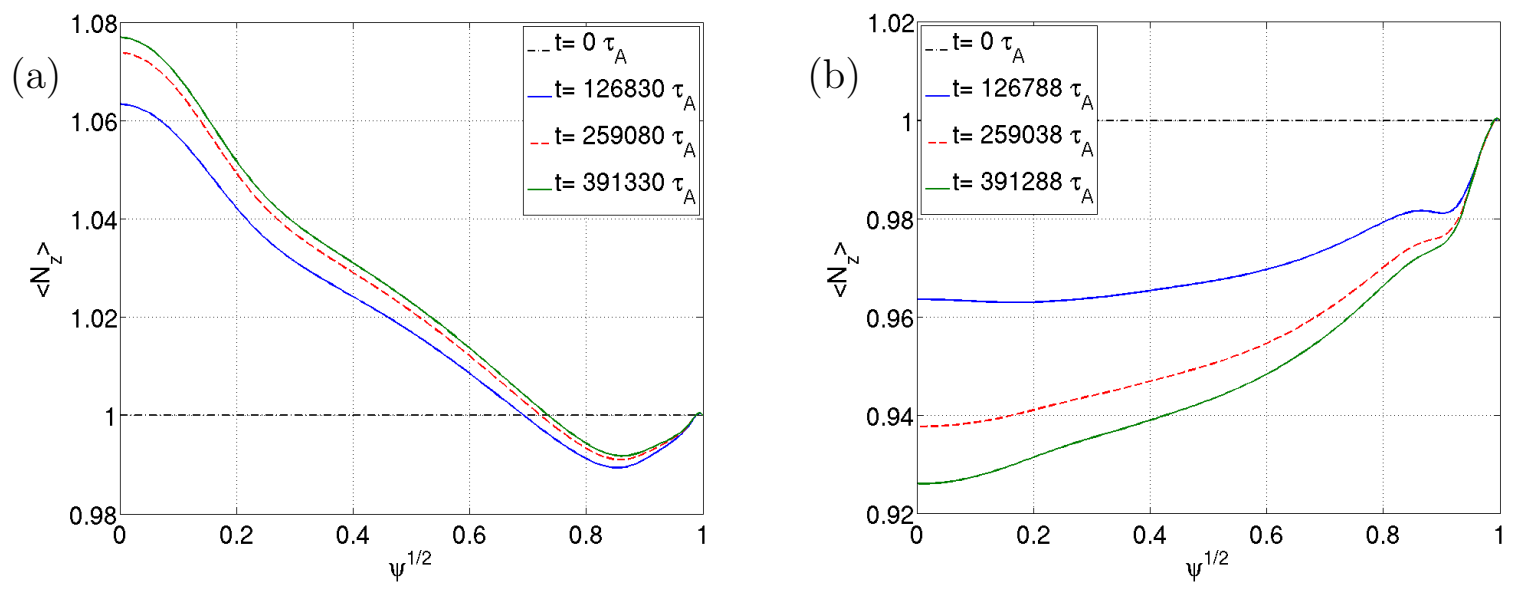

Figure 3. Evolution of the impurity profiles for the cases (a) without thermal force $\left(C_{0}=0\right)$, and (b) with an appropriate coefficient for the thermal force $\left(C_{0}=3 / 2\right)$ as predicted by the neoclassical theory. The temperature screening effect appears in presence of the thermal force.

\subsection{Effects of sawtooth oscillations on impurity behaviour}

When the rescaled plasma parameters described in Section 3.2 are used, sustained sawtooth cycles are obtained with the XTOR-2F code (Fig.4). These simulations are used to assess the impurity behaviour in sawtoothing plasmas. The neoclassical time scales are very large compared to the duration of sawtooth crashes. Hence, the temperature screening effect, resulting from the collisions between the main ion species and the impurity, affects the impurity dynamics only during the sawtooth ramp phase.

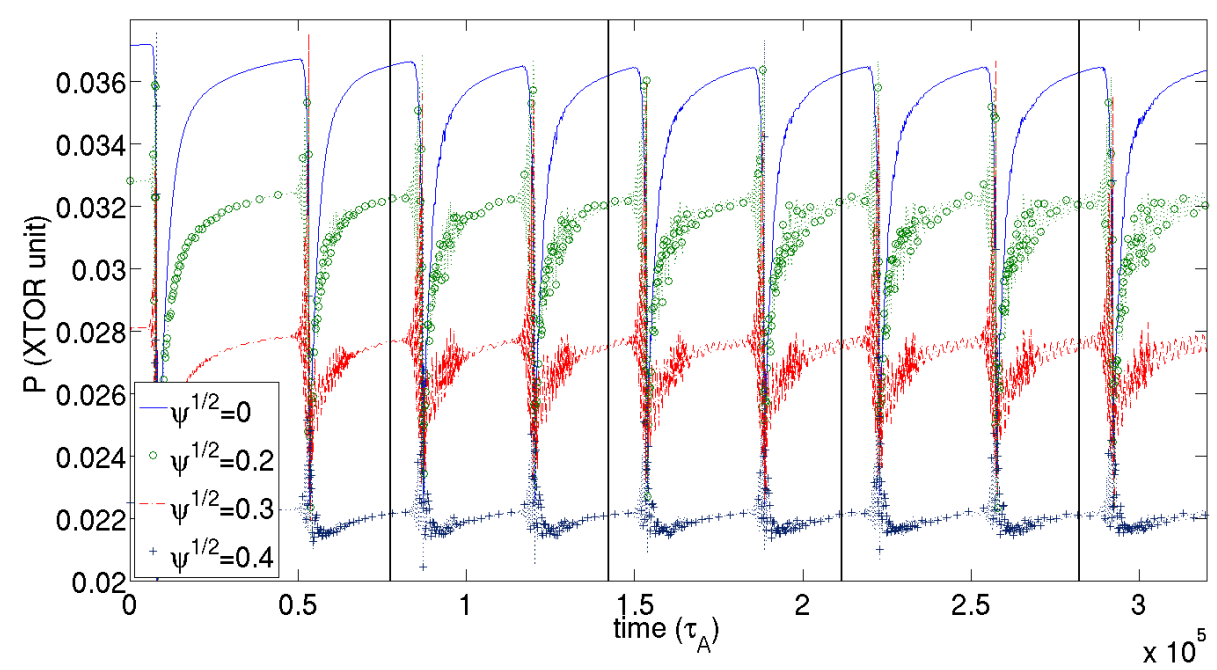

Figure 4. Evolution of the pressure at different radii. The time points which correspond to the surfaces averaged impurity profiles in Fig. 5 are marked by the vertical lines. These time points are chosen during the quiescent phase where the plasma has reached a quasi-stationary equilibrium. 


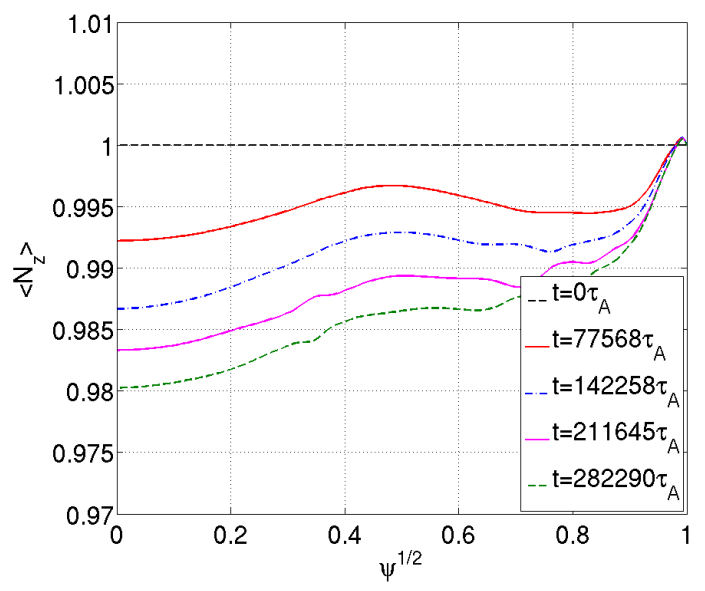

Figure 5. Evolution of the surface averaged impurity density throughout the sawtooth cycles. The profiles correspond to the times marked by the vertical lines in Fig.4.

The evolution of the surface averaged impurity profiles throughout the sawtooth cycles is presented in Fig.5. The impurity profiles are those between the post-cursor and precursor oscillations of a sawtooth cycle. It is noticed again that when the temperature screening effect is taken into account, an impurity outflow is driven throughout the sawtooth cycles. However, a clear difference on the impurity behaviour is seen between the simulations with and without sawteeth (Fig.6). The surface averaged impurity profiles after $t \simeq 3.2 \times 10^{5} \tau_{A}$ show that the impurity outflux is smaller in the presence of sawteeth. Indeed, the central impurity density has decreased by about $2 \%$ for the case with sawteeth, which is less than a third of what is obtained for the case without sawteeth.

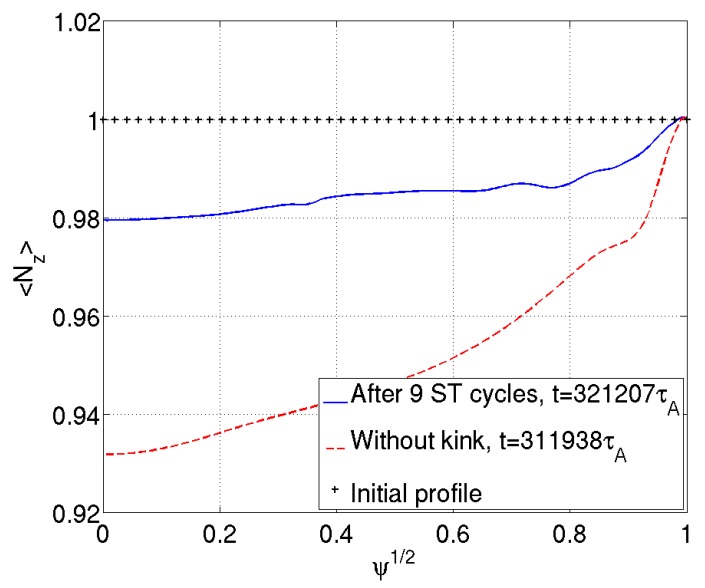

Figure 6. Comparison of the surface averaged impurity density profiles between the cases with (solid line) and without (dashed line) sawteeth after $t \simeq 3.2 \times 10^{5} \tau_{A}$ from the initial profile $(+)$. The presence of sawtooth cycles decreased the quantity of impurity expelled from the core region. 

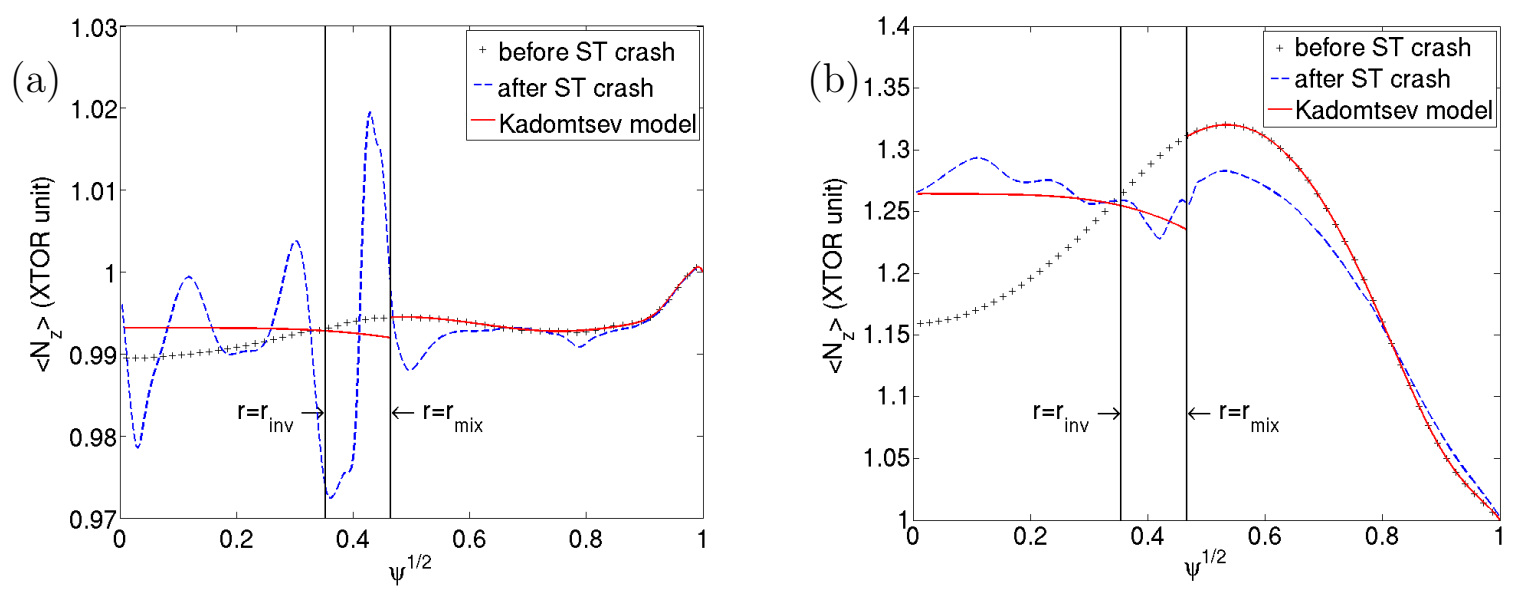

Figure 7. Impurity profiles after sawtooth crash for (a) lowly-contrasted and (b) highly-contrasted pre-crash profiles $(+)$. Post-crash impurity profiles computed by the XTOR-2F code (dashed lines) and with Kadomtsev reconnection model (solid lines) are shown. The inversion radius $r_{i n v}$ and the mixing radius $r_{m i x}$ are marked by vertical lines. For a pre-crash profile with more contrasted hollowness, the penetration of impurity is clearly visible and is in accordance with the Kadomtsev reconnection model.

The weakened temperature screening effect is partly related to time averaged temperature profile which is less peaked than the initial temperature profile. This is due to a periodic flattening of the temperature profile at sawtooth crashes [22]. Hence, the overall radial pinch velocity of the impurity is less important than the case without sawteeth, where the temperature profile remains unchanged. Also, the particle redistribution during the sawtooth crash tends to weaken the impurity outflow. Indeed, sawtooth crashes drive an efficient radial transport of impurities. In particular, the Kadomtsev reconnection model [23],[24] predicts an influx of impurity during sawtooth crashes for a hollow pre-crash impurity profile [25], regardless its contrast (Fig.7). However, in the XTOR-2F simulations, a density perturbation of about $5 \%$ is found in the impurity profiles after a sawtooth crash (blue dashed lines). Impurity penetration is not visible as predicted by the Kadomtsev reconnection model, if the pre-crash impurity density profile is less contrasted than the fluctuation (Fig.7a). On the contrary, the impurity profile after a sawtooth crash obtained by the XTOR-2F code is consistent with the prediction for a well-contrasted pre-crash profile (Fig.7b). The radial redistribution of particles due to sawtooth crashes is sufficient to weaken the outward impurity flux compared to the case without sawteeth, although the impurity profiles in the present study are almost flat.

\section{Discussion}

In this paragraph, the mechanisms that underlie the impurity transport during a crash are investigated in more details. For this purpose, different time points during a 
sawtooth crash are selected and marked by vertical lines in Fig.8a. The corresponding magnetic configurations are displayed as Poincaré plots in Figs.8b-f. In Fig.9, the impurity density that corresponds to the time points marked in Fig.8a are shown in color background. As the electron mass is very small compared to the ion mass, electron heat conduction is expected to be faster than the ion convection. Thus, the temperature contours are often considered as a representation of magnetic surfaces. Unlike for the
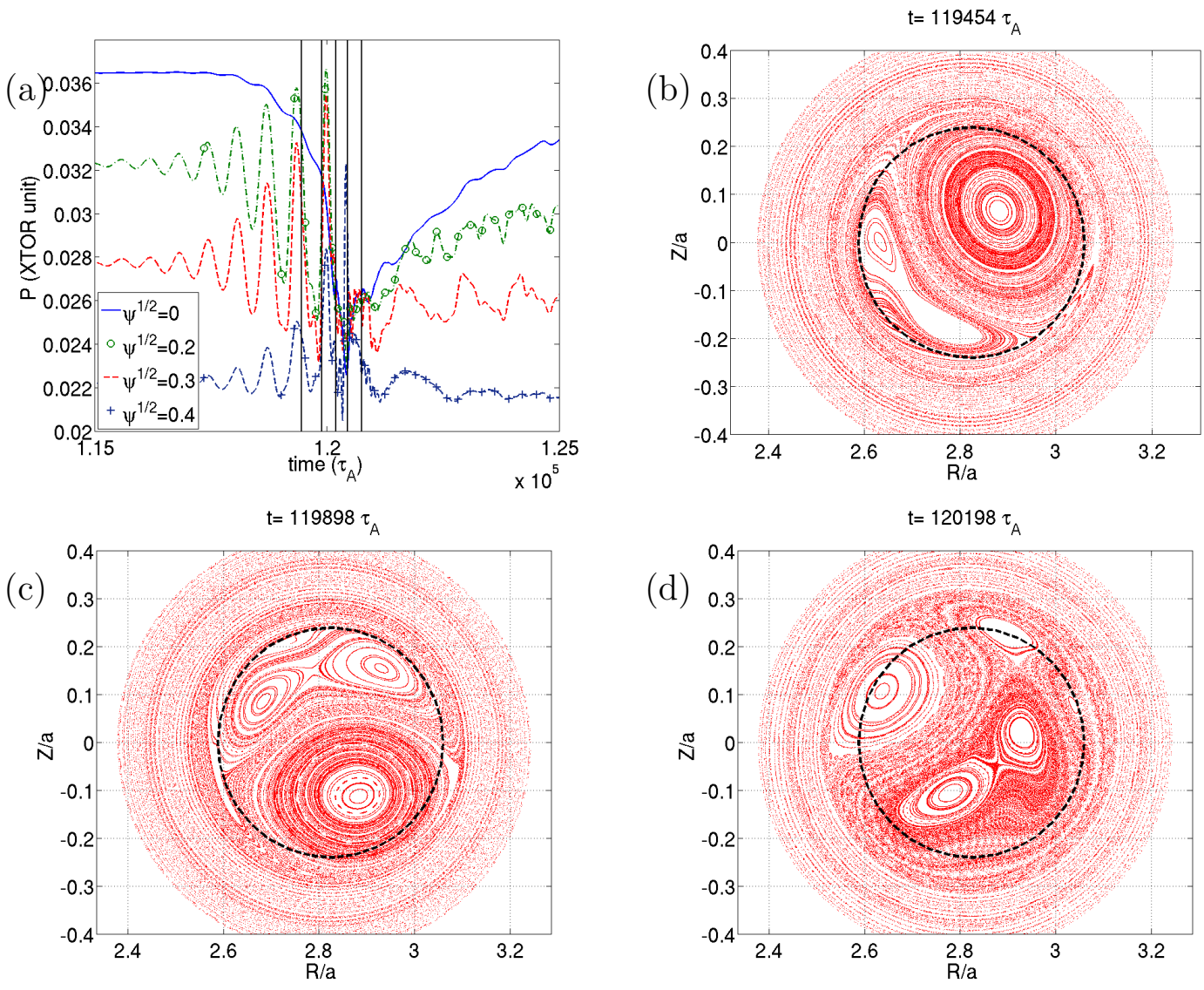

$t=120453 \tau_{A}$
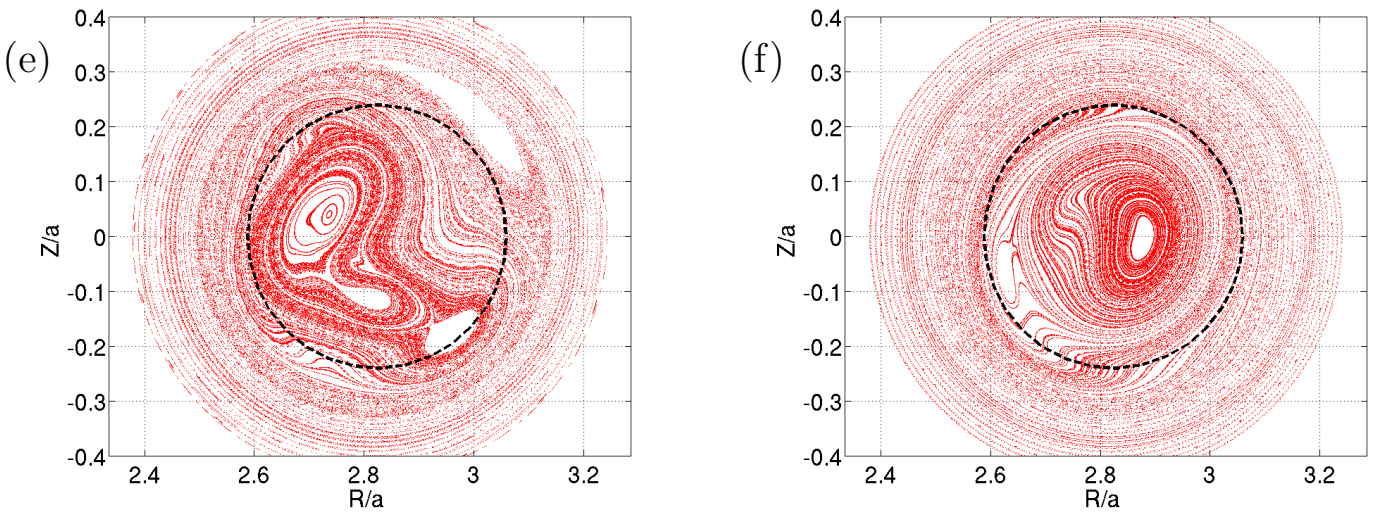

Figure 8. (a) Time evolution of pressure at different radii during sawtooth crash and (b)-(f) Poincaré plots corresponding to the time points marked by vertical lines. The $q=1$ surface is represented by dashed lines. 

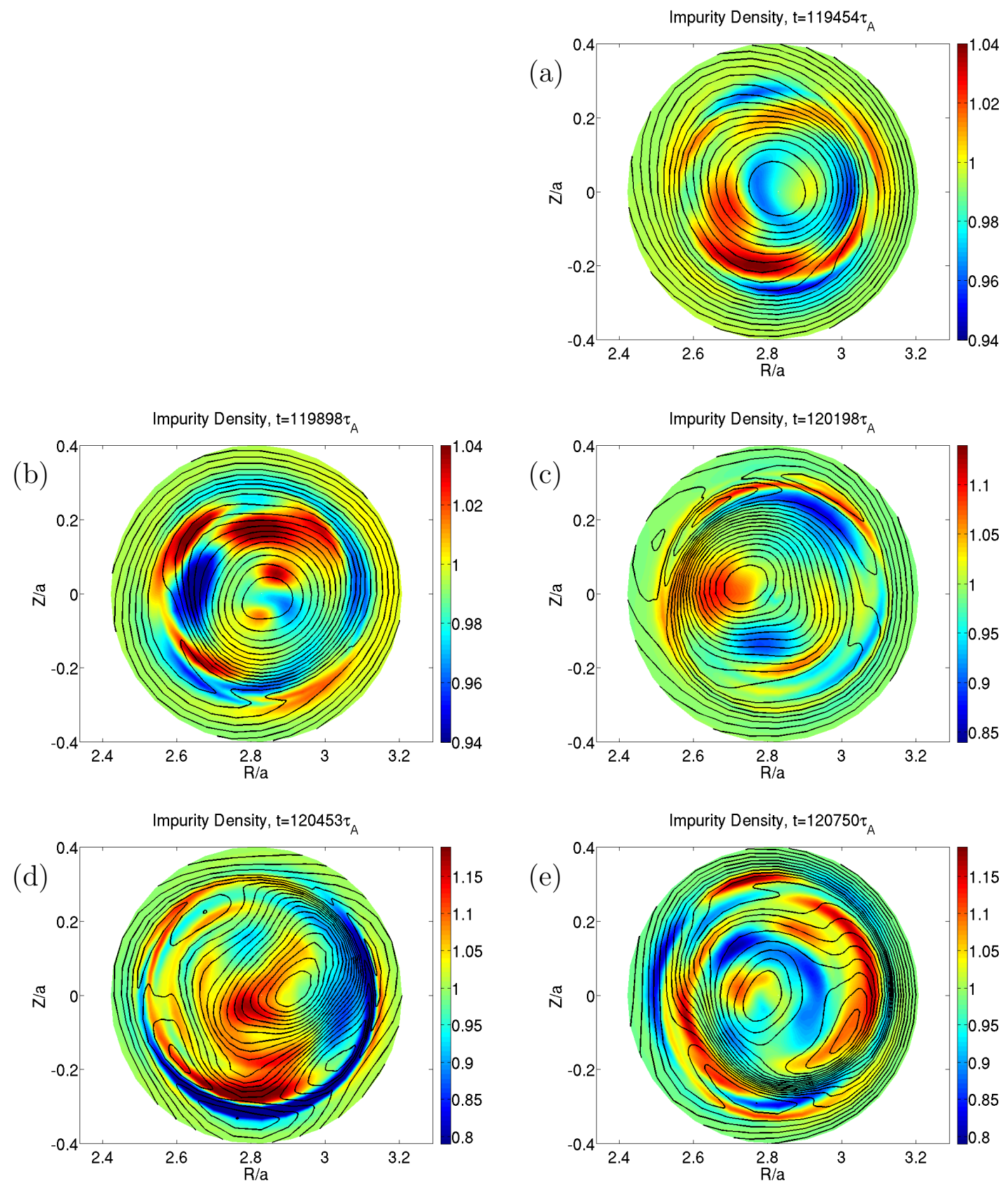

Figure 9. Impurity density structures during the sawtooth crash in the background and the electric potential $\phi$ in contour lines. Each figure corresponds to Poincaré plots of Fig.8.

ion species, the convection perpendicular to magnetic surfaces also has to be considered. The contour lines which represent the reconstructed stream function associated with the $\mathbf{E} \times \mathbf{B}$ velocity are also shown in Fig.9. This is an indication of the parallel flux role, which tends to align the impurity density structures with the magnetic topology. It can also be seen that the orientation of these structures is reminiscent of the $\mathbf{E} \times \mathbf{B}$ velocity field. 

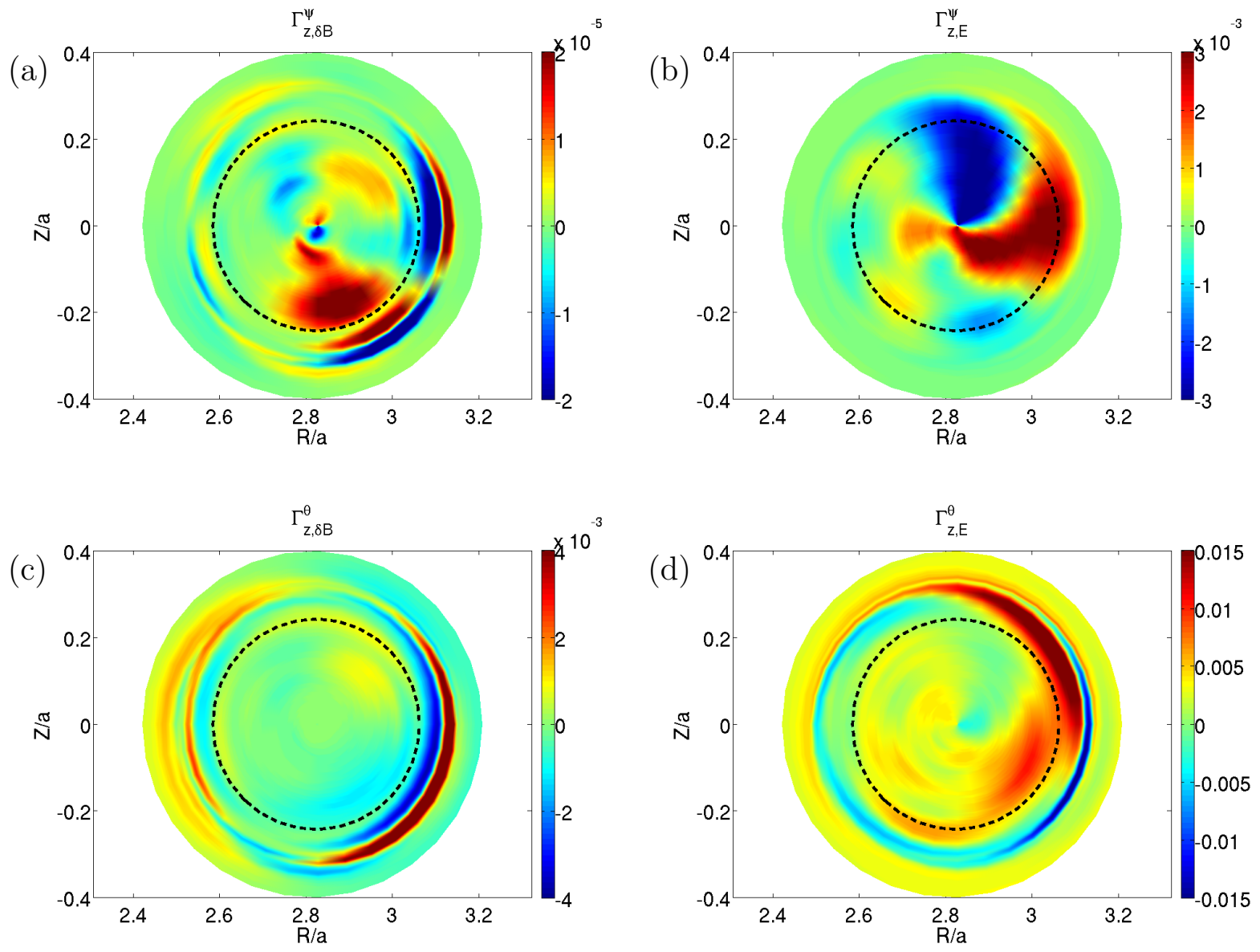

Figure 10. The radial and poloidal components $(\mathrm{a}) \Gamma_{z, \delta B}^{\psi},(\mathrm{b}) \Gamma_{z, E}^{\psi},(\mathrm{c}) \Gamma_{z, \delta B}^{\theta}(\mathrm{d}) \Gamma_{z, E}^{\theta}$ of the parallel impurity flux $\boldsymbol{\Gamma}_{z, \delta B}=N_{z} V_{z, \|} \mathbf{B} / B$ and the impurity flux driven by the $\mathbf{E} \times \mathbf{B}$ velocity $\boldsymbol{\Gamma}_{z, E}=N_{z} \mathbf{V}_{E}$, corresponding to $t=120453 \tau_{A}$ are represented in the poloidal plane at $\varphi=0$. The dashed lines represent the $q=1$ surface.

It can be noticed that the impurity density structures bear similarities with the magnetic configuration with a slight poloidal shift. At the same time, these structures are driven by the $\mathbf{E} \times \mathbf{B}$ flow. For example, in Fig.9b, the impurity density bump and hole located respectively at $\theta \simeq \pi / 2$ and $\theta \simeq \pi$, are more or less similar to magnetic surfaces in the island (Fig.8c). These same structures are then rotated along the reconstructed streamlines (Figs.9b,c).

In order to deduce the quantitative correlations of the impurity density structures with magnetic flutter and $\mathbf{E} \times \mathbf{B}$ convection, it is necessary to compare the two components of the impurity flux. From the expression (3) of the velocity, the impurity fluxes due to field line bending and $\mathbf{E} \times \mathbf{B}$ drift respectively reads

$$
\Gamma_{z, \delta B}=N_{z} V_{z, \|} \mathbf{B} / B
$$

and

$$
\Gamma_{z, E}=N_{z} \mathbf{V}_{E}
$$



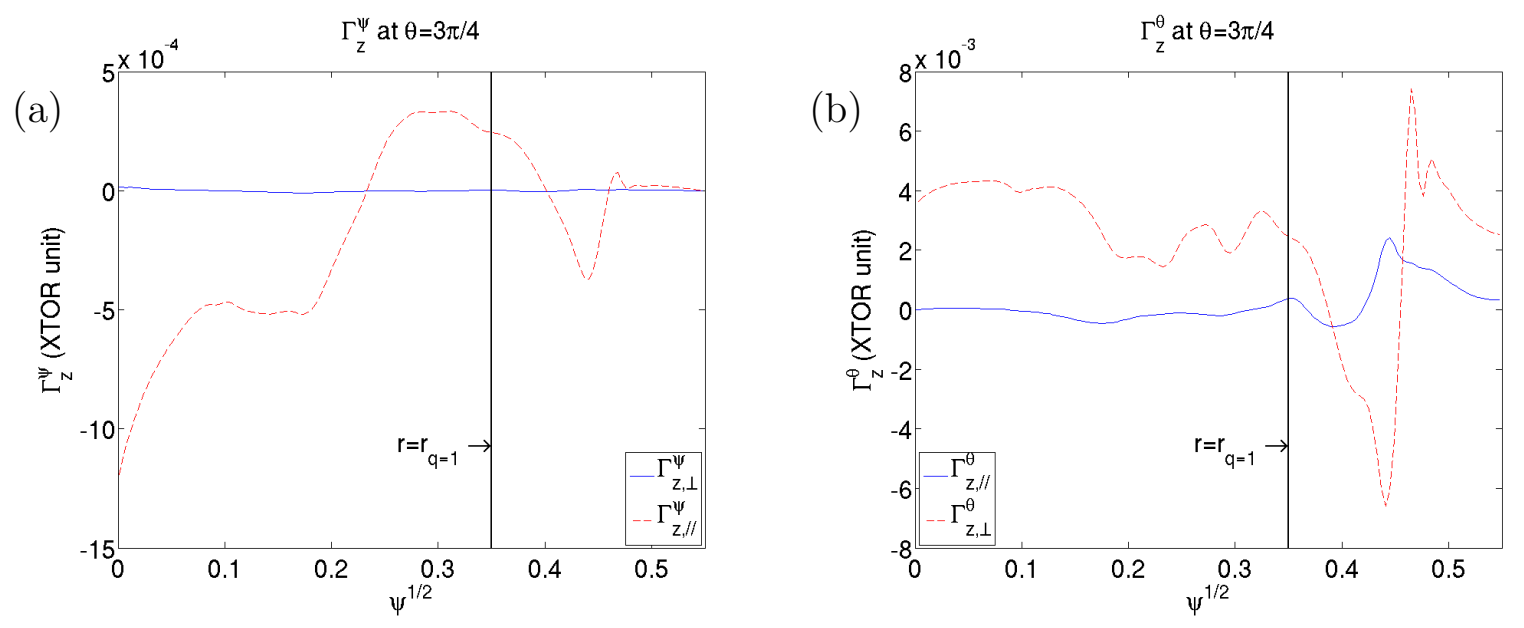

Figure 11. The profiles at $(\theta, \varphi)=(3 \pi / 4,0)$ of the (a) radial and (b) poloidal components of the impurity flux along the magnetic field line $\boldsymbol{\Gamma}_{z, \delta B}$ (solid lines) and the flux driven by $\mathbf{E} \times \mathbf{B}$ velocity $\boldsymbol{\Gamma}_{z, E}$ (dashed lines). The $q=1$ radius is marked by vertical lines.

The impurity flux driven by the diamagnetic velocity is neglected since $Z \gg 1$ for heavy impurities. In Fig.10, the radial and poloidal components $\left(\Gamma_{z, \delta B}^{\psi}, \Gamma_{z, \delta B}^{\theta}\right)$ and $\left(\Gamma_{z, E}^{\psi}, \Gamma_{z, E}^{\theta}\right)$ in the poloidal section at $\varphi=0$, are represented, by defining

$$
\Gamma_{z, y}^{x} \equiv \Gamma_{z, y} \cdot \nabla x
$$

These fluxes are calculated at time $t=1.2 \times 10^{5} \tau_{A}$ (Fig.10), when the magnetic reconnection process during sawtooth crash has almost ended. At this time, the expelled hot core is located outside the $q=1$ surface, and the cooler island occupies a large part of the core region (Fig.8e). Also, complex impurity density structures are seen inside the $q=1$ surface (Fig.9d). It appears that $\Gamma_{z, E}^{\psi} \gg \Gamma_{z, \delta B}^{\psi}$ and $\Gamma_{z, E}^{\theta} \gg \Gamma_{z, \delta B}^{\theta}$ in most of the core region, i.e. the $\mathbf{E} \times \mathbf{B}$ flux overcomes the magnetic flutter flux in all directions. However, in some specific locations, e.g. $\pi / 2<\theta<\pi$ and outside the inversion radii, $\Gamma_{z, E}^{\psi} \simeq \Gamma_{z, \delta B}^{\psi}$ and $\Gamma_{z, E}^{\theta} \simeq \Gamma_{z, \delta B}^{\theta}$ (Figs.10,11). Hence, the impurity structures during the sawtooth crash are driven by the two fluxes, but the $\mathbf{E} \times \mathbf{B}$ velocity plays a prominent role in most locations during sawtooth crashes.

In Ref.[37], soft X-ray (SXR) and temperature measurements are analysed in detail during a sawtooth crash. The presence of the background metal impurities allows the impurity behaviour to be deduced from SXR emission. It is shown that a SXR emission peak is expelled out of the $q=1$ surface during a sawtooth crash, along with the hot core. However, SXR and temperature contours become uncorrelated after the sawtooth crash. Particles escape from the reconnection layer during the sawtooth crash and are transported with their respective thermal velocity. Therefore, the impurity transport time scale is large compared to the electron one which can be associated with the modifications of the magnetic configuration. The impurity behaviour during a sawtooth crash obtained by the XTOR-2F code is consistent with this picture. Indeed, the impurity density structures are not representative of magnetic flux surfaces, and 
they are spread along streamlines associated with the $\mathbf{E} \times \mathbf{B}$ velocity.

As a consequence, the impurity density bump and hole result from the time lag between impurity and electron transport.

\section{Conclusion}

Fluid equations for impurities in the Pfirsch-Schlüter regime have been implemented in the XTOR-2F code in order to investigate the combined effect of neoclassical transport and sawtooth crashes. An analytical calculation shows that the neoclassical impurity flux in the Pfirsch-Schlüter regime is recovered by adjusting the friction between the impurity and the main ion species.

It has been numerically verified that the impurity radial fluxes obtained with this model are consistent with neoclassical predictions. Simulations show that the presence of sawtooth crashes changes the impurity thermal screening. Indeed, the time averaged outward impurity flux is weakened in presence of sawteeth. This is mainly due to a sudden influx of impurity during the sawtooth crash for hollow pre-crash profiles. Also, periodic flattening of the core temperature profile weakens the thermal screening effect during the recovery phase. Impurity density perturbation in the percent range persist after each sawtooth crash. This makes it difficult to assess the agreement with the predictions of the Kadomtsev model for weakly hollow impurity profiles. Nevertheless, the long time scale behaviour is definitely consistent with a weakening of the thermal screening effect.

The impurities penetrate inside the $q=1$ surface through impurity density bump and hole which appear during sawtooth crashes. These structures are shown to be mainly driven by the $\mathbf{E} \times \mathbf{B}$ velocity in the core region. Impurities are clustered in the form of structures as they are redistributed from the reconnection layer with a thermal velocity which is smaller than the electron thermal velocity.

\section{Acknowledgments}

This work was granted access to the HPC resources of Aix-Marseille Université financed by the project Equip@Meso (ANR-10-EQPX-29-01). This work was also carried out in the framework of GENCI project 056348 and using the HELIOS supercomputer system (IFERC-CSC), Aomori, Japan, under the Broader Approach collaboration, implemented by Fusion for Energy and JAEA.

\section{Appendix A. Poloidal asymmetry of impurity density}

With the expressions (9), (14), (17) and the constraint (19), the parallel friction force reads, in the small inverse aspect ratio limit $\varepsilon=r / R_{0} \ll 1$,

$\mathcal{R}_{\|, z i}=m_{z} N_{z, 0} \nu_{z i} \frac{T_{0}}{e_{z}}\left(\partial_{\psi} \ln P_{z, 0}-\frac{e_{z}}{e_{i}} \partial_{\psi} \ln P_{i, 0}+C_{0} \frac{e_{z}}{e_{i}} \partial_{\psi} \ln T_{i, 0}\right) 2 r \cos \theta$ 
by writing $B=B_{0} /(1+\varepsilon \cos \theta)$ and $I=B_{0} R_{0}$. In this limit, the parallel gradient is written as $\nabla_{\|}=1 /\left(q R_{0}\right) \partial_{\theta}$.

If the poloidal asymmetry of impurity density is only considered, the parallel projection of the force balance equation (18) reads

$$
\frac{1}{q R_{0}} \frac{\partial_{\theta} N_{z}}{N_{z, 0}}=-\frac{2 q}{R_{0}} \frac{\nu_{z i}}{\omega_{c, z}}\left[\frac{R_{0}}{L_{N_{z}}}+\frac{e_{z}}{e_{i}} \frac{R_{0}}{L_{N_{i}}}-\left(C_{0}-1\right) \frac{e_{z}}{e_{i}} \frac{R_{0}}{L_{T_{i}}}\right] \cos \theta
$$

with $\omega_{c, z} \equiv e_{z} B_{0} / m_{z}$ is the cyclotron frequency of the impurity and $L_{Y} \equiv\left|\partial_{r} \ln Y\right|^{-1}$ is the gradient length of the quantity $Y$.

The integration of the expression (A.2) along $\theta$ for heavy impurities $\left(e_{z} / e_{i} \gg 1\right)$ gives

$$
\frac{N_{z, 1}}{N_{z, 0}}=-2 q^{2} \frac{e_{z}}{e_{i}} \frac{\nu_{z i}}{\omega_{c, z}}\left[\frac{R_{0}}{L_{N_{i}}}-\left(C_{0}-1\right) \frac{R_{0}}{L_{T_{i}}}\right] \sin \theta
$$

Hence, the ordering used to derive the Pfirsch-Schlüter fluxes in Section 2 can be written as

$$
2 q^{2} \frac{e_{z}}{e_{i}} \frac{\nu_{z i}}{\omega_{c, z}}\left|\frac{R_{0}}{L_{N_{i}}}-\left(C_{0}-1\right) \frac{R_{0}}{L_{T_{i}}}\right| \ll 1
$$

It should be noted that despite the small parameter $\delta_{z}=\nu_{z i} / \omega_{c, z} \ll 1$, steep gradients of the ion density and temperature profiles can be sufficient to break the assumption on small poloidal asymmetry due to the high charge numbers of heavy impurities.

\section{References}

[1] Pitts R et al 2011 J, Nucl. Mater. 415 S957

[2] Noda N, Philipps V and Neu R 1997 J. Nucl. Mater. 241-243 227

[3] Pütterich T, Neu R, Dux R, Whiteford A D, O'Mullane M G, Summers H P and the ASDEXUpgrade Team 2010 Nucl. Fus. 50025012

[4] Rice J E et al 2002 Nucl. Fus. 42510

[5] Leigheb M et al 2007 Plasma Phys. Control. Fusion 491897

[6] Neu R et al 2007 Plasma Phys. Control. Fusion 49 B59

[7] Fedorczak N et al 2015 J. Nucl. Mater. 46385

[8] Hinton F L and Wong S K 1985 Phys. Fluids 283082

[9] Catto P J, Bernstein I B and Tessarotto M 1987 Phys. Fluids 302784

[10] Belli E A, Candy J and Angioni C 2014 Plasma Phys. Control. Fusion 56124002

[11] Hirshman S P and Sigmar D J 1981 Nucl. Fus. 211079

[12] Helander P and Sigmar D J 2005 Collisional Transport in Magnetized Plasmas (Cambridge : Cambridge Univ. Press)

[13] Angioni C et al 2014 Nucl. Fus. 54083028

[14] Casson F J et al 2015 Plasma Phys. Control. Fusion 57014031

[15] Romanelli M and Ottaviani M 1998 Plasma Phys. Control. Fusion 401767

[16] Fülop T and Helander P 1999 Phys. Plasmas 63066

[17] Angioni C and Helander P 2014 Plasma Phys. Control. Fusion 56124001

[18] Hender T C et al 2016 Nucl. Fus. 56066002

[19] Sertoli M, Odstrcil T, Angioni C and the ASDEX-Upgrade Team 2015 Nucl. Fus. 55113029

[20] Dux R, Peeters A G, Gude A, Kallenbach A, Neu R and the ASDEX-Upgrade Team 1999 Nucl. Fus. 391509 
[21] Yamada I, Yamazaki K, Oishi T, Arimoto H and Shoji T 2010 Plasma Fusion Res. 5 S1022

[22] Porcelli F, Boucher D and Rosenbluth M N 1996 Plasma Phys. Control. Fusion 382163

[23] Kadomtsev B B 1975 Sov. J. Plasma Phys. 1389 [1975 Fiz. Plasmy 1 710]

[24] Furno I et al 2001 Nucl. Fus. 41403

[25] Ödblom A, Anderson D, Eriksson L-G and Lisak M 1996 Phys. Plasmas 3956

[26] Nicolas T, Lütjens H, Luciani J-F, Garbet X and Sabot R 2014 Phys. Plasmas 21012507

[27] Lütjens H and Luciani J-F 2010 J. Comp. Phys. 2298130

[28] Hinton F L and Hazeltine R D 1976 Rev. Mod. Phys. 48239

[29] Hazeltine R D and Meiss J D 2003 Plasma Confinement (Mineola, NY : Dover Publications Inc.)

[30] Braginskii S I 1965 Rev. of Plasma Phys. 1205

[31] Rutherford P H 1974 Phys. Fluids 71782

[32] Nicolas T, Sabot R, Garbet X, Lütjens H, Luciani J-F, Guimarães-Filho Z, Decker J and Merle A 2012 Phys. Plasmas 19112305

[33] Halpern F D, Lütjens H and Luciani J-F 2011 Phys. Plasmas 18102501

[34] Ahn J-H et al 2016 Phys. Plasmas 23052509

[35] Mattioli M, De Michelis C and Pecquet A L 1998 Nucl. Fus. 381629

[36] Guirlet R et al 2010 Nucl. Fus. 50095009

[37] Nagayama Y et al 1991 Phys. Rev. Lett. 673527 